\title{
A self-consistent upward leader propagation model
}

\author{
Marley Becerra and Vernon Cooray \\ Division for Electricity and Lightning Research, Ångström Laboratory, Uppsala University, \\ Sweden, SE 751 21, Box 534, Uppsala, Sweden \\ E-mail: Marley.Becerra@angstrom.uu.se
}

Received 21 May 2006, in final form 28 June 2006

Published 4 August 2006

Online at stacks.iop.org/JPhysD/39/3708

\begin{abstract}
The knowledge of the initiation and propagation of an upward moving connecting leader in the presence of a downward moving lightning stepped leader is a must in the determination of the lateral attraction distance of a lightning flash by any grounded structure. Even though different models that simulate this phenomenon are available in the literature, they do not take into account the latest developments in the physics of leader discharges. The leader model proposed here simulates the advancement of positive upward leaders by appealing to the presently understood physics of that process. The model properly simulates the upward continuous progression of the positive connecting leaders from its inception to the final connection with the downward stepped leader (final jump). Thus, the main physical properties of upward leaders, namely the charge per unit length, the injected current, the channel gradient and the leader velocity are self-consistently obtained. The obtained results are compared with an altitude triggered lightning experiment and there is good agreement between the model predictions and the measured leader current and the experimentally inferred spatial and temporal location of the final jump. It is also found that the usual assumption of constant charge per unit length, based on laboratory experiments, is not valid for lightning upward connecting leaders.
\end{abstract}

(Some figures in this article are in colour only in the electronic version)

\section{Introduction}

As a lightning downward stepped leader approaches the ground, upward discharges develop from protruding grounded objects such as trees, Franklin rods or sharp corners in buildings. These upward discharges, called connecting leaders, propagate towards the downward moving leader trying to make connection with it. When any upward leader successfully intercepts the downward stepped leader, a conductive path is created through which the high lightning current is drained to the ground. In order to avoid any damage produced by the lightning flash to the struck object, the place where the successful upward connecting leader is initiated is designed to safely conduct the lightning current to the ground. Thus, guard wires and Franklin rods are usually used to intercept downward stepped leaders and protect grounded structures such as power transmission lines and buildings against the harmful lightning currents. Each guard wire and Franklin rod has a given lightning protection zone limited by the maximum lateral distance that the initiated upward leader can propagate to attach the downward stepped leader before it strikes either the ground or other grounded point [1].

In order to evaluate the lightning protection zone for isolated masts and transmission lines, Eriksson [2,3] proposed a pioneering method which evaluates the inception and propagation of the upward connecting leader. He used the critical radius concept [4] to compute the distance between the tip of the downward stepped leader and the structure when an upward leader is initiated from it. This distance is usually referred to as the striking distance [2]. In addition, he considered that the connection of the downward leader with the newly initiated upward leader depends on the relative velocities of both leaders, and the interception is only possible within a defined geometric volume. The lateral extension of 
this volume is defined by the interception of the parabolic loci defined by the downward/upward leader velocity ratio (taken as unity by Eriksson) and the striking distance. Thus, if the stepped leader enters into this collection volume, it is then assumed that there is a successful connection between the two leaders.

Later, Dellera and Garbagnati [5, 6] proposed a more sophisticated method of analysis compared with Eriksson's, which involves the simulation of the propagation of both leaders based on electrostatic calculations. The progression of the leaders is modelled by finite line charges and the direction of each following segment is assumed to be given by the maximum electric field vector in front of it. The relative velocity of the leaders is varied between 4 and 1 , but no details were given about the way this variation was implemented. The charge per unit length of the upward leader was assumed to be constant and equal to $50 \mu \mathrm{C} \mathrm{m}^{-1}$ from laboratory experiments, whereas the downward leader charge was estimated as a function of the prospective return stroke current.

On the other hand, Rizk [7,8] suggested an iterative process to determine the maximum lateral distance of the downward leader where the connection with an upward leader initiated from a tall grounded structure takes place. For a given lateral distance of a downward leader, the initiation of the upward leader from the studied structure was computed with his own criterion $[9,10]$. Then, the propagation of the upward leader is followed by assuming a unit downward/upward leader velocity ratio with a velocity vector directed towards the tip of the unperturbed downward leader propagating towards the ground. The connection between both leaders is decided when the mean potential gradient between the tips of the leaders is equal to the streamer gradient taken as $500 \mathrm{kV} \mathrm{m}^{-1}$. The upward connecting leader was modelled as a channel with constant average gradient from a semi-empirical expression for leaders under switching impulses.

Because of lack of knowledge available at the time these models were proposed, they used different leader inception models and assumed different upward leader properties as well as different velocity ratios. Consequently, large differences are found between the predictions obtained from each model. Nevertheless, there is nowadays better knowledge of the physical parameters involved in the propagation of upward leaders. This fact is partly due to the experiments conducted at instrumented tall structures $[11,12]$ and the use of triggered lightning techniques [13-16] and partly due to the improvement in theoretical leader inception and propagation models [17-19].

Thus, the results of different experiments with natural and triggering lightning suggest that it is not possible to generalize the behaviour of upward connecting leaders as in $[2,3,5-8]$. For instance, it has been observed that the temporal evolution of the upward leader velocity in lightning triggered experiments does not follow a well-defined pattern but instead changes from flash to flash [13]. Also, there are some doubts about the validity of using parameters of leaders obtained in laboratory to describe upward connecting leaders. For these reasons, a better leader progression model should be capable of self-consistently estimating the physical properties of upward leaders. These include the charge per unit length, the injected current, the leader channel gradient and the velocity of the upward leader under the influence of a downward moving leader. With this objective in mind, a physical model to simulate the initiation and propagation of positive upward connecting leaders from grounded structures is introduced in this paper. Initially, the model evaluates the initiation of upward connecting positive leaders considering the dynamic conditions imposed by the descent of the downward leader [20]. These conditions include the time variation of the electric field produced by the stepped leaders and the space charge associated with streamers and aborted leaders produced before the stable leader inception takes place. Once the upward leader is incepted, the model self-consistently simulates its propagation based on the analysis of the total charge of the corona streamer zone in front of the leader tip. In this way, the leader velocity as well as the injected current and the charge per unit length of leader are calculated as it propagates towards the downward stepped leader. The model results are validated by comparing its predictions with the data obtained in an altitude triggered lightning experiment [14].

\section{Physics of the upward leader propagation model}

In order to theoretically analyse the propagation of positive connecting leaders under the influence of down-coming stepped leaders, it is necessary to use self-consistent models both for the inception and for the propagation of the positive leader. Bondiou-Clergerie et al [21] presented a onedimensional simulation of the main physical leader processes which was applied to a triggered rocket experiment. However, the set of equations that were used to derive the leader and corona front velocities, the leader current and other important physical parameters were not given.

Recently, Becerra and Cooray [19] introduced a selfconsistent leader inception model based on the physics of the conversion of streamer to leader [17, 18,22]. In [20], they also implemented their model to dynamically evaluate the inception of upward positive leaders considering the time variation in the electric field produced by the stepped leaders and the space charge associated with streamers and aborted leaders produced before the stable leader inception takes place. In this paper, the Becerra and Cooray leader model $[19,20]$ is extended and improved for the study of the advancement of the upward connecting leader up to the final jump.

At the beginning of the analysis, the streamers that occur before the leader inception takes place are modeled as in [20]. If the energy input supplied by the streamers is high enough to increase the temperature of the stem above a critical value (around $1500 \mathrm{~K}$ [22]), the first leader segment is created (unstable leader inception). This transition from streamer to leader takes place if the total charge $\Delta Q$ in the second or successive corona bursts is equal or larger than about $1 \mu \mathrm{C}$ [22] after at least one corona burst (first corona). Then, the leader starts propagating with corona streamers developing at its tip, which sustain the thermal transition and advancement of the leader head [22].

Thus, the simulation of the leader propagation is started by evaluating the first leader segment and the corona charge in front of it. The model uses the electrostatic representation of the corona zone in front of the leader channel tip proposed 

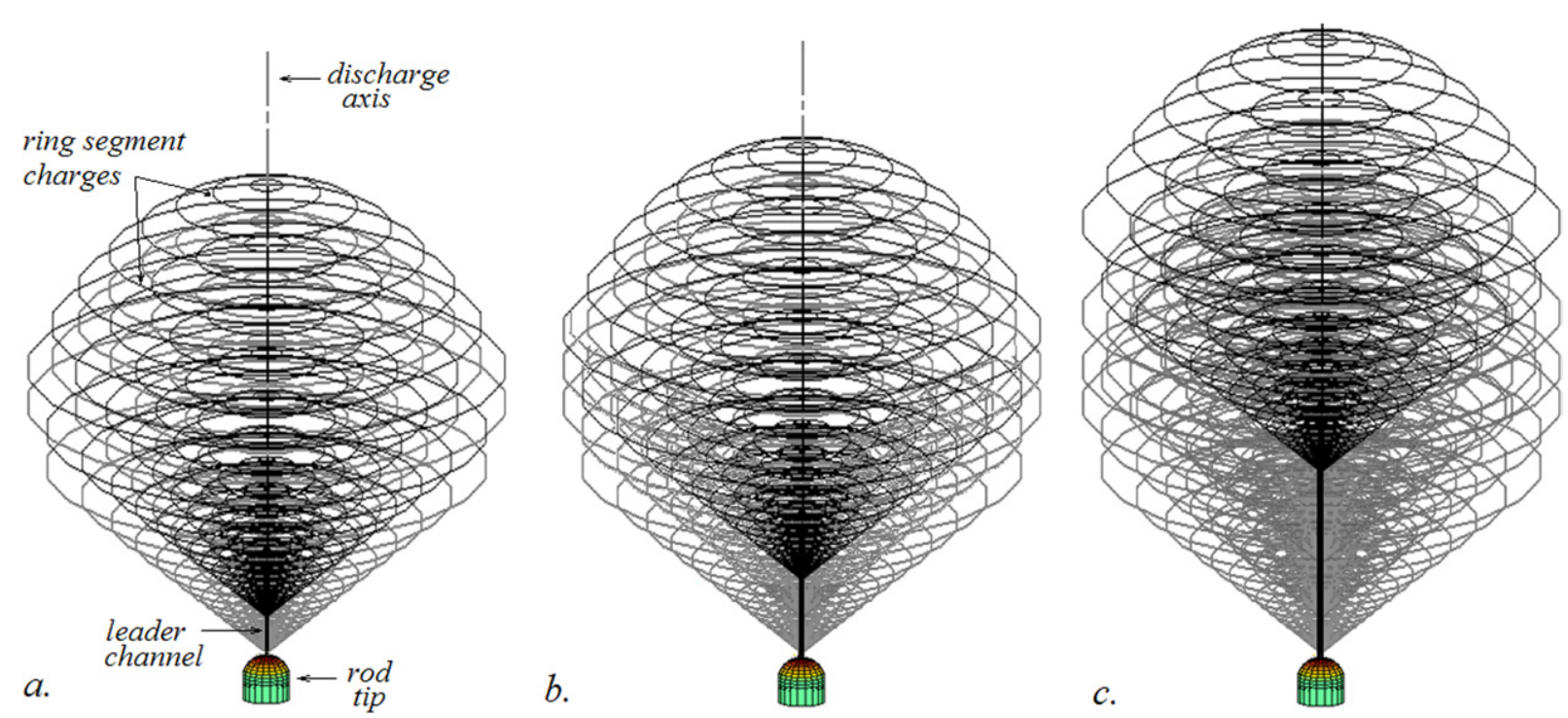

Figure 1. CSM modelling of the upward leader propagation: $(a),(b)$ and $(c)$ show the corona zone charges in front of the leader channel as the leader propagates in the presence of the previous space charge around the discharge axis.

by Goellian et al [18]. This representation assumes that the streamers created from the tip of the leader channel have a constant potential gradient $E_{\mathrm{str}}$ across the corona zone. Then, the charge simulation method (CSM) [23] is used to compute the total corona charge required to satisfy this condition, assuming that the streamers split into many branches defining a conical volume [24]. The leader channel is represented by finite lines with increasing length and a point charge at its tip, while the corona streamer zone is modelled using generalized ring segment charges of uniform charge density as is shown in figure 1. A detailed explanation of the CSM calculation can be found in [19].

Once the corona charge of the first leader segment is computed, the lengths of the following newly created leader segments $\left(l_{\mathrm{L}(i)}\right.$ for $\left.i=2,3, \ldots\right)$ are computed using the constant relation between leader current $I_{\mathrm{L}}$ and velocity $v_{\mathrm{L}}$ proposed by Bondiou and Gallimberti [17]:

$$
v_{\mathrm{L}}=\frac{I_{\mathrm{L}}}{q_{\mathrm{L}}}
$$

where $q_{\mathrm{L}}$ is the charge per unit length necessary to achieve the transition from the streamer corona to a new leader segment in the active region in front of the leader channel. It assumes that the advancement of the leader (i.e. the leader velocity) is determined by the current collected by the leader head due to the streamer filaments converging into it. This current determines the energy input at the leader head for the transition from streamers to a new filamentary leader channel [17]. The leader velocity is proportional to the current through the parameter $q_{\mathrm{L}}$ which represents the charge per unit length required to thermalize a new leader segment. Hence, the position of the leader head during each simulation step $l_{\mathrm{L}(\mathrm{i})}$ is computed from equation (1) as

$$
l_{\mathrm{L}(i)}=l_{\mathrm{L}(i-1)}+\frac{\Delta Q(t-\Delta t)}{q_{\mathrm{L}}},
$$

where $\Delta Q(t-\Delta t)$ is the total corona charge at the front of the leader tip during the previous simulation time step.
The total corona charge $\Delta Q(t)$ during the following simulation steps is computed with the CSM calculation, considering the leader tip potential and the potential produced by the ring charges used to model the preceding corona zones during previous steps. In order to improve the calculation of the leader tip potential presented in [19], the thermohydrodynamical model of the leader channel proposed by Gallimberti [22] is used here. The Gallimberti's model relates the gradient along the leader channel directly to the injected charge through it. Thus, the radius $a_{\mathrm{L}(i)}$ and electric field $E_{\mathrm{L}(i)}$ of each $i$ th leader segment produced during each simulation time step $t$ are computed as follows:

$a_{\mathrm{L}(i)}(t)$

$=\sqrt{a_{\mathrm{L}(i)}^{2}(t-\Delta t)+\frac{(\gamma-1) \cdot E_{\mathrm{L}(i)}(t-\Delta t) \cdot \Delta Q(t-\Delta t)}{\pi \cdot \gamma \cdot p_{0}}}$,

$E_{\mathrm{L}(i)}(t)=E_{\mathrm{L}(i)}(t-\Delta t) \cdot \frac{a_{\mathrm{L}(i)}^{2}(t-\Delta t)}{a_{\mathrm{L}(i)}^{2}(t)}$,

where $p_{\mathrm{o}}$ is the atmospheric pressure and $\gamma$ is the ratio between the specific heats at constant volume and constant pressure. In this way, a more reliable value for the potential and radius of the leader is obtained during its propagation towards the downward stepped leader, in comparison with the results obtained with the semi-empirical equation of Rizk [9] used in [19]. The continuous propagation of the upward leader can be modelled with the previous equations and by considering the parameters shown in table 1 .

At this point, most of the input parameters of the model are quite well-known quantities except the charge per unit length $q_{\mathrm{L}}$. Since the propagation characteristics of the leader are strongly influenced by $q_{\mathrm{L}}$ (see equation (2)), a discussion on the physical meaning of this parameter and how to extract it from the available data is warranted here.

Essentially, the charge per unit length $q_{\mathrm{L}}$ is inversely correlated with the amount of energy transferred into heat from 
Table 1. Parameters used for the presented physical leader propagation model.

\begin{tabular}{|c|c|c|c|c|}
\hline Sym & Description & Value & Units & Ref \\
\hline$L_{\mathrm{L}}^{(1)}$ & Initial leader length & $2 \times 10^{-2}$ & $\mathrm{~m}$ & - \\
\hline$a_{\mathrm{L}}$ & $\begin{array}{l}\text { Initial channel radius } \\
\text { of a newly created } \\
\text { leader segment }\end{array}$ & $10^{-3}$ & $\mathrm{~m}$ & [24] \\
\hline$r_{\text {Ltip }}$ & $\begin{array}{l}\text { Initial leader tip curvature } \\
\text { radius of a newly created } \\
\text { leader segment }\end{array}$ & $2.5 \times 10^{-5}$ & $\mathrm{~m}$ & [22] \\
\hline$E_{\text {str }}$ & Positive streamer gradient & $4.5 \times 10^{5}$ & $\mathrm{~V} \mathrm{~m}^{-1}$ & [22] \\
\hline$E_{\mathrm{L}}$ & $\begin{array}{l}\text { Potential gradient before } \\
\text { a new leader segment } \\
\text { is created }\end{array}$ & $4.5 \times 10^{5}$ & $\mathrm{~V} \mathrm{~m}^{-1}$ & [22] \\
\hline
\end{tabular}

the total energy available in front of the leader tip. The more energy at the leader tip is converted into thermal energy, the less charge from the corona zone is required to thermalize a new leader segment. The total energy available in front of the leader tip is stored in the translational, rotational, electronical and vibrational reservoirs [22], and it depends mainly upon the potential and radius of the leader tip. The fraction of that total energy which is effectively converted into heat is affected by the humidity content and the available time to transfer that energy [22]. In this way, the energy transfer is more effective with high humidity and low leader velocity, becoming less efficient as the leader speeds up.

In long gap experiments, the charge per unit length $q_{\mathrm{L}}$ was initially defined as the ratio between the injected charge and the length of the leader channel. It is also measured as an average proportionality factor between the real velocity and current during the propagation of positive leaders in long gaps [24-26]. The average values for the charge $q_{\mathrm{L}}$ obtained in laboratory range from 20 to $50 \mu \mathrm{C} \mathrm{m}^{-1}$. The variation in the parameter is mainly due to the differences in humidity and the risetime of the applied voltage, the latter defining the leader tip potential. Since the potential of the leader tip remains approximately constant when a voltage with critical time to crest is applied to a long gap [22], the charge per unit length $q_{\mathrm{L}}$ is usually taken as a constant value during the leader propagation.

As for natural lightning, the values of $q_{\mathrm{L}}$ obtained in laboratory may be applicable only at times close to the initiation of the upward connecting leaders when the leader is not more than a few metres long. However, as the upward leader extends over many tens of metres, the value of $q_{\mathrm{L}}$ may deviate from the laboratory values. This is because the tip potential of an upward leader under the influence of a downward leader is continuously increasing as it propagates while the leader tip potential in laboratory sparks remains approximately constant [22]. Moreover, the axial velocity of leaders in long sparks does not exceed $3 \times 10^{4} \mathrm{~m} \mathrm{~s}^{-1}$, while the velocity of the upward leader has been measured between $10^{4}$ and $1.2 \times 10^{6} \mathrm{~m} \mathrm{~s}^{-1}[13,27]$. Due to the fact that the charge per unit length $q_{\mathrm{L}}$ depends upon both the potential and velocity of the leader, it does not seem correct to take values of $q_{\mathrm{L}}$ for lightning studies uniquely based on long gap experiments.

Therefore, it is necessary to make estimates of this parameter from experiments with triggered or natural lightning. Lalande [28] calculated the average positive leader charge per unit length during an altitude triggered lightning experiment as $65 \mu \mathrm{C} \mathrm{m}^{-1}$. Nonetheless, he estimated values of $q_{\mathrm{L}}$ close to $200 \mu \mathrm{C} \mathrm{m}^{-1}$ during the late stage of propagation of the triggered upward leader. In addition, a rough estimation of the charge per unit length $q_{\mathrm{L}}$ under natural conditions can be obtained from the positive upward leaders measured in Mount San Salvatore [27]. These upward leaders propagate with velocities ranging from $2 \times 10^{4}$ to $3 \times 10^{5} \mathrm{~m} \mathrm{~s}^{-1}$ [27], with increasing continuous current up to about few hundred amperes [29]. Then, if the ratio between the leader current and its velocity at the end of the leader propagation is calculated, a value of $q_{\mathrm{L}}$ larger than around $500 \mu \mathrm{C} \mathrm{m}^{-1}$ can be estimated, which is at least ten times larger than the ones measured in long gaps.

However, due to scanty experimental evidence of the values of the charge per unit length $q_{\mathrm{L}}$, of upward leaders under natural conditions, a theoretical analysis seems to be the best way to obtain physically reasonable values for this parameter. In [22,24], Gallimberti proposed a thermodynamic analysis of the transition region where the corona converges to the leader tip in order to estimate $q_{\mathrm{L}}$. It assumes that the creation of a new leader segment takes place when the temperature rise in the transition zone is high enough to produce thermal detachment of negative ions. According to this theory, the charge per unit length $q_{\mathrm{L}}$ required to achieve the transition to a new leader segment can be estimated as

$$
q_{\mathrm{L}}=\frac{I_{\mathrm{L}}}{K \cdot\left(f_{\mathrm{ert}}+f_{v} \cdot\left(\frac{\tau_{1}}{\tau_{1}+\tau_{v t}}\right)\right) \cdot \int_{l_{\mathrm{L}}}^{l_{t}}(J \cdot E) \mathrm{d} l},
$$

where $I_{\mathrm{L}}$ is the leader current, $f_{\text {ert }}$ is the fraction of the energy transferred into electronic, rotational and translational excitation and $f_{v}$ is the fraction of the collision energy transferred into the vibrational reservoir. The term $\tau_{1} /\left(\tau_{1}+\tau_{v t}\right)$ represents the fraction of the vibrational energy which can be relaxed into thermal energy during the leader transition time $\tau_{1}$ in relation to the vibrational relaxation time $\tau_{v t}$. The integral term in the denominator corresponds to the specific power available in the transition zone. It is defined by the product of the current density $J$ and the average electric field $E$ across the transition zone $\Delta l_{1}$ defined between the leader tip $l_{\mathrm{L}}$ and a point $l_{t}$ where the specific power becomes negligible. In this way, the thermal energy is released in the transition zone during the leader transit time $\tau_{1}=\Delta l_{1} / v_{\mathrm{L}}$, where $v_{\mathrm{L}}$ is the leader velocity. This leads to an increase in the temperature in front of the leader until the transition from corona to the new leader channel segment takes place. The parameter $K$ is a constant that involves the critical temperature required for the transition and the density of neutrals in the transition zone.

By representing the leader channel as a paraboloid with tip curvature radius of $25 \mu \mathrm{m}$, Gallimberti analytically computed the specific power in front of the leader tip and estimated $q_{\mathrm{L}}$ under laboratory conditions, in agreement with measured values. In this paper, equation (5) is used to check the physical validity of an exponential-like equation chosen beforehand as an estimate of $q_{\mathrm{L}}$ as a function of the computed leader velocity for the case of upward connecting leaders. Thus, during each simulation step the value of $q_{\mathrm{L}}$ is taken from this equation for the current computed leader velocity $v_{\mathrm{L}}$ and is used in equation (2). In addition, the specific power input at the tip of the leader 


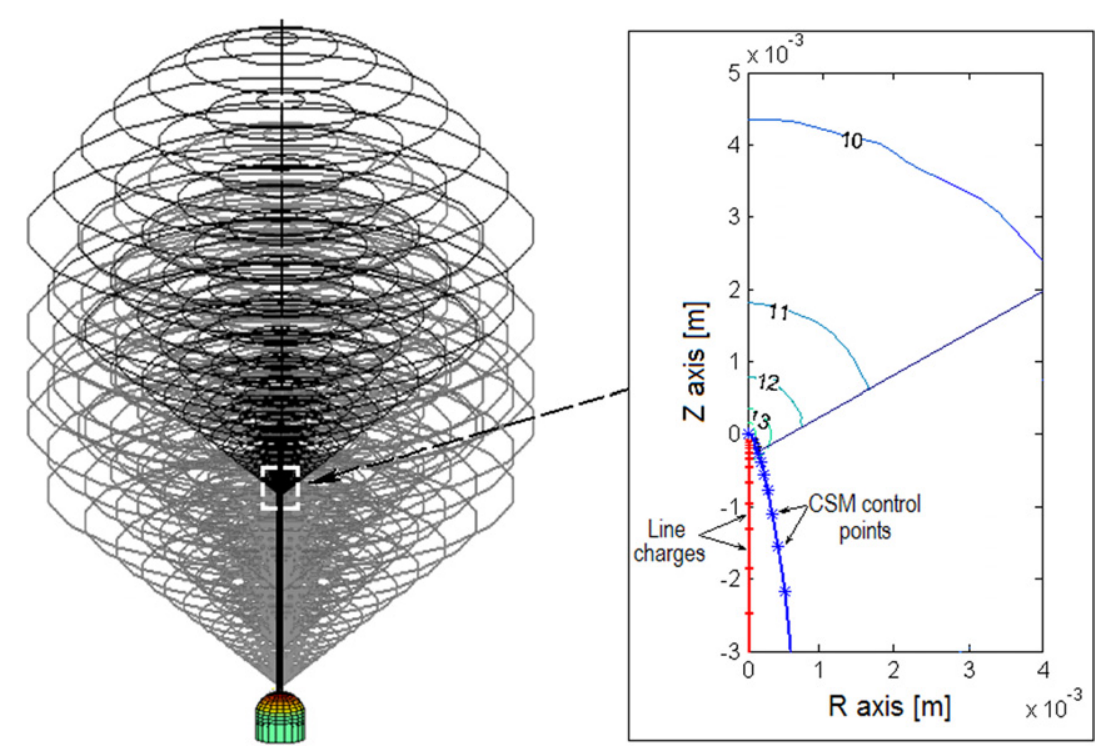

Figure 2. Details of the CSM representation of the leader channel tip and the specific power in the corona zone. The contours correspond to the logarithm of the specific power evaluated also accounting for the effect of the space charge produced during previous steps.
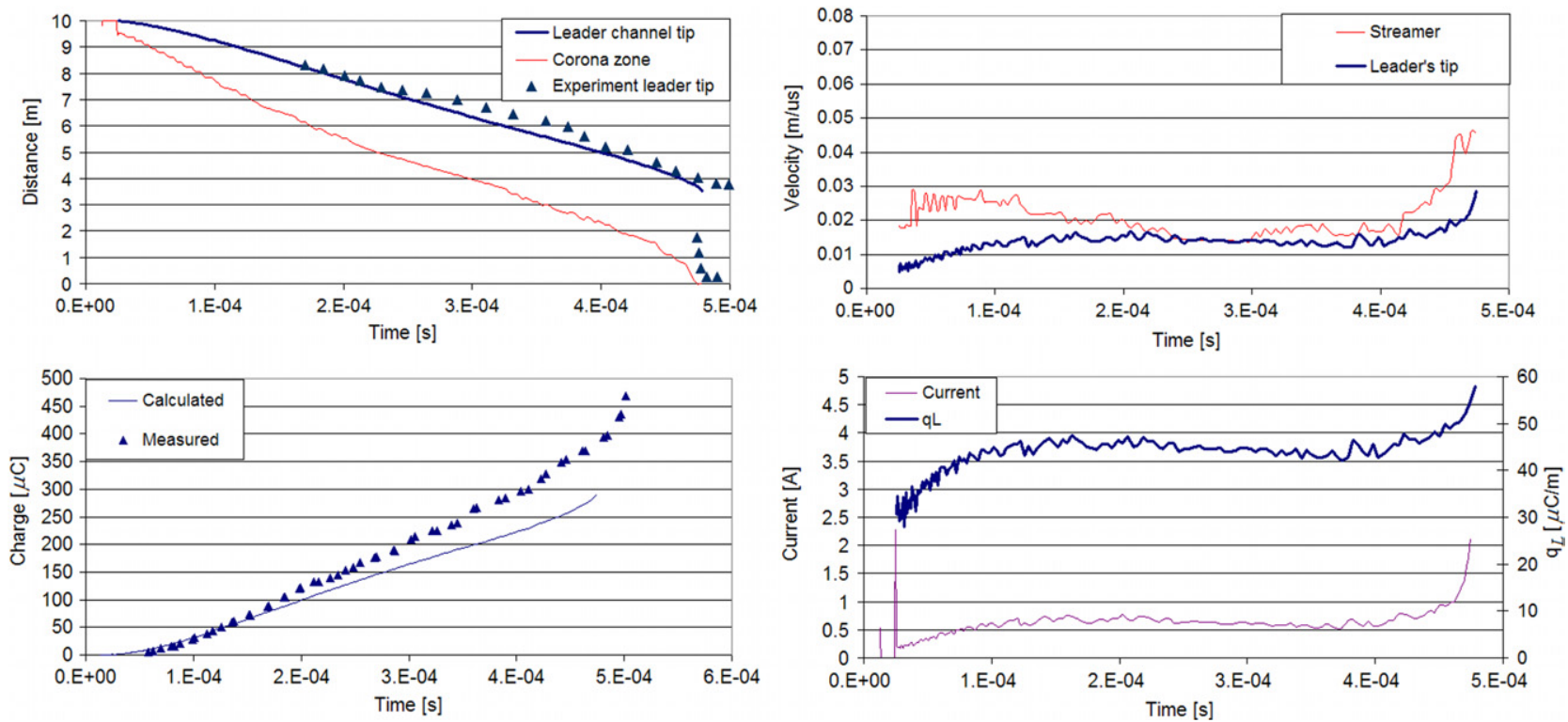

Figure 3. Computed discharge characteristics for a $10 \mathrm{~m}$ long cone to a plane configuration subjected to a $1850 \mathrm{kV}$ switching impulse with $500 \mu$ s time to crest. (a) streak image, $(b)$ leader and streamer tip velocity, $(c)$ injected charge, $(d)$ leader current and charge per unit length. The measured values [25] of the leader tip and the injected charge are also shown for sake of comparison.

channel is computed and equation (5) is evaluated. If at the end of the simulation the values of the assumed $q_{\mathrm{L}}$ and the ones computed with equation (5) throughout the analysis are not in good agreement, a different equation is chosen accordingly and the full simulation is repeated.

During the evaluation of equation (5) it is assumed that all the energy is transferred into vibrational excitation $\left(f_{\text {ert }}=0\right.$, $f_{v}=1$ ) and that the current density $J$ is approximately equal to the ratio of the leader current $I_{\mathrm{L}}$ by the surface of the transition zone at each radial distance from the leader tip. A sketch of the CSM representation of the leader tip and the specific power in the corona zone is shown in figure 2. The vibrational relaxation time $\tau_{v t}$ is taken as $100 \mu \mathrm{s}$ [22] and the value of the constant $K$ is set in such a way that the value of $q_{\mathrm{L}}$ computed with (5) is equal to $65 \mu \mathrm{C} \mathrm{m}^{-1}$ [29] when the leader velocity reaches $2 \times 10^{4} \mathrm{~m} \mathrm{~s}^{-1}$.

\section{Application of the model to simulate leaders in laboratory experiments}

In order to perform a first check of the validity of the model, the propagation of a leader in a $10 \mathrm{~m}$ long cone to a plane gap subjected to a switching impulse voltage of $1850 \mathrm{kV}$ with $500 \mu \mathrm{s}$ time to crest is simulated. In the simulation the charge per unit length $q_{\mathrm{L}}$ is computed as a function of the leader velocity according to the equation presented in [28].

Figure 3 shows the temporal and spatial development of the leader channel in the gap (streak image), leader and 
streamer velocities, the injected charge into the gap and the leader current predicted by the model. In the same figure the measured values of the leader tip position and the injected charge [25] are also depicted for comparison purposes. Note the good agreement between the measured and computed values for the streak image and the injected charge.

Similarly, the present model is used to evaluate the propagation of leaders for the $10 \mathrm{~m}$ long gap electrodic configurations reported in [24]. Hence, a hemisphere electrode with a diameter of $0.1 \mathrm{~m}$ and sphere electrodes with diameters of 0.6 and $1 \mathrm{~m}$ are simulated under the $600 \mu$ s time to crest switching breakdown voltage. Since streak images of these experiments are not reported in [24], only the computed times to breakdown are compared with the measured values. In all the cases, there is good agreement between the computed and measured times to breakdown (with differences lower than $15 \%)$.

\section{Propagation of upward leaders: a study case}

Unfortunately, there are still not many experiments in the literature that can be used to fully understand the propagation parameters of upward leaders under natural conditions. This is partly due to the fact that in most of the field experiments $[13,16,27]$ not all the important parameters such as the leader current, the background electric field and the leader speed have successfully been measured simultaneously. To the best of our knowledge, only in the altitude triggered lightning experiment performed by Lalande et al [14] the leader propagation current (lower than $100 \mathrm{~A}$ ), the background electric field change (measured at $50 \mathrm{~m}$ from the wire) and the leader luminosity (with static and streak photography) were measured simultaneously. In this experiment, a rocket first spools out $50 \mathrm{~m}$ of grounded wire, followed by $400 \mathrm{~m}$ of insulating Kevlar and from it to the rocket tail a second (triggering) copper wire [14]. In this technique a positive leader is first initiated from the top end of the upper floating wire and consequently a negative leader is initiated from its bottom end. In response to this downward moving negative leader, an upward moving connecting positive leader is initiated from the grounded bottom wire section. The data from this experiment are used to test the validity of the present model.

In this experiment, the inception of the upward leader occurred when the electric field change at the ground level at a point located $50 \mathrm{~m}$ from the wire reached $10 \mathrm{kV} \mathrm{m}^{-1}$, while the connection point of the two leaders was located about $20 \mathrm{~m}$ from the grounded wire tip. During the triggered flash, the upward leader did not propagate more than a few tens of metres before reaching the final jump state and therefore the experimental data refers only to the early stage of the positive leader propagation.

To test the validity of model predictions, a similar scenario is simulated and the results obtained are compared with the measured leader propagation parameters. In the analysis, the grounded wire segment is simulated by a hemispherically capped grounded rod, while the downward leader is assumed to be a uniform line charge located above the rod and extending downwards from a point located at $450 \mathrm{~m}$ above the ground level. The average velocity of the downward leader is taken
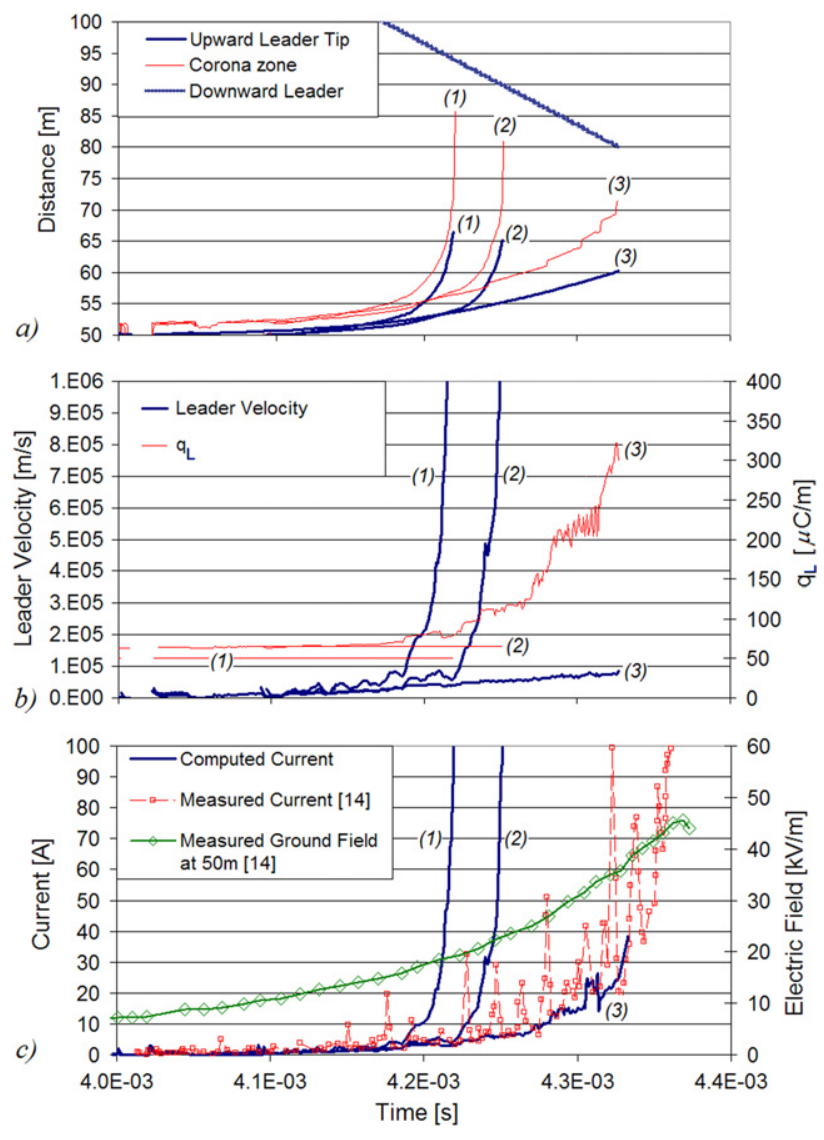

Figure 4. Discharge characteristics for the upward leader during the altitude triggered lightning experiment 9516 [14] computed assuming a constant $q_{\mathrm{L}}$ equal to $50 \mu \mathrm{C} \mathrm{m}^{-1}$ (case 1), $65 \mu \mathrm{C} \mathrm{m}^{-1}$ (case 2) and taking it as a function of the leader velocity according to Gallimberti's equation (case 3). (a) Streak image, (b) leader velocity and $q_{\mathrm{L}}$ and $(c)$ computed and measured leader current and measured ground electric field at $50 \mathrm{~m}$ from the wire. The time scale is initiated at the moment when the floating conductor reaches $450 \mathrm{~m}$ above ground.

as $1.3 \times 10^{5} \mathrm{~m} \mathrm{~s}^{-1}$ when the height of its tip is about $120 \mathrm{~m}$ above the ground [14]. The leader charge is computed in such a way that the leader field at the ground level corresponds to the measured electric field change. Since the static background electric field produced by the thundercloud during the experiment was not reported, a typical value of about $15 \mathrm{kV} \mathrm{m}^{-1}$ is taken from the electric field profile measured by Willet et al [15] at an altitude of $50 \mathrm{~m}$.

Figure 4 shows the computed streak image, the velocity and current of the upward leader in the altitude triggering experiment, simulated by considering three different cases. The first case assumes a constant charge per unit length $q_{\mathrm{L}}$ equal to $50 \mu \mathrm{C} \mathrm{m}^{-1}$ (case 1), which is used by Dellera and Garbagnati [5,6] for their leader progression model. The second case takes a constant value of $q_{\mathrm{L}}$ equal to $65 \mu \mathrm{C} \mathrm{m}^{-1}$ (case 2) calculated by Lalande [29] and the latter assumes a changing $q_{\mathrm{L}}$ as a function of the leader velocity which is validated with equation (5) (case 3 ).

As can be clearly seen, when the charge per unit length $q_{\mathrm{L}}$ is considered as a constant average value during the propagation of the upward leader (cases 1 and 2), the leader velocity increases drastically, taking unrealistic values larger 


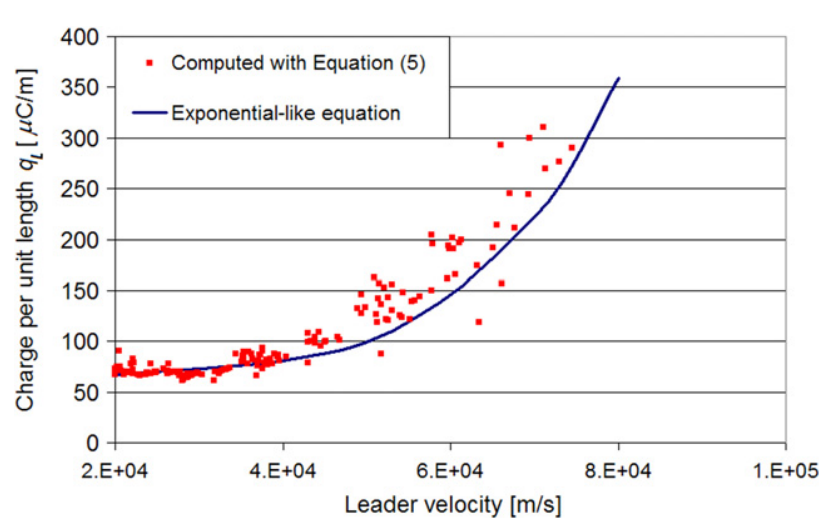

Figure 5. Simulated values of the charge per unit length $q_{\mathrm{L}}$ for the altitude triggered lightning experiment 9516 [14] and the equation used to correlate the velocity and the current of the leader.

than $10^{6} \mathrm{~m} \mathrm{~s}^{-1}$ (figure $4(b)$ ). This causes the simulated final jump to take place much quicker and at a larger altitude than that observed (at $4.37 \mathrm{~ms}$ and at about $70 \mathrm{~m}$ above ground [14]). The disagreement between these cases and the experiment is because a constant $q_{\mathrm{L}}$ does not reflect the physical fact that an increase in the leader velocity reduces the efficiency of energy transfer into heat which in turn increases the amount of charge required to create a new leader segment. Hence, the simulated leader will deliberately speed up without maintaining the balance between the leader velocity and the electrostatic energy available in front of the leader.

On the other hand, note the excellent agreement between the computed and measured currents as well as the good agreement with the time and position of the final jump for case 3. A difference with cases 1 and 2 where a constant value for $q_{\mathrm{L}}$ is assumed, in this case the predicted time of the final jump (at $4.33 \mathrm{~ms}$ ), is similar to the value observed experimentally (at $4.37 \mathrm{~ms}$ ). Furthermore, the computed leader velocity increases reasonably from about $10^{4}$ to $8 \times 10^{4} \mathrm{~m} \mathrm{~s}^{-1}$ before the final jump takes place. These results clearly show that the charge per unit length $q_{\mathrm{L}}$ of the upward leader cannot be assumed as a constant value and that instead it increases during the propagation of the leader. In this way, the increment in the electrostatic energy at the upward leader tip produced by the descent of the downward leader is balanced by the increase in the charge per unit length $q_{\mathrm{L}}$, keeping the upward leader velocity within physically reasonable values, which are also in agreement with the field observations.

This fact suggests that a more physical and proper evaluation of the attachment of the downward leader by the upward leader is obtained when $q_{\mathrm{L}}$ is self-consistently computed by the leader propagation model. Figure 5 shows the comparison of the final obtained relationship between $q_{\mathrm{L}}$ and the computed leader velocity and the values computed by equation (5) for the altitude triggering experiment discussed in this paper. It is important to point out that the obtained values of $q_{\mathrm{L}}$ shown in figure 5 apply only for the conditions present in the triggered lightning experiment considered here. Thus, the values of the main properties of upward connecting leader (namely the charge per unit length $q_{\mathrm{L}}$ and the leader velocity) under the influence of a lightning downward stepped leader with a given prospective return stroke peak current and average velocity, may differ from those computed in this paper. This is because the conditions at the tip of an upward moving leader depend on the electrostatic conditions imposed by the downward stepped leader. Consequently, different values of the charge required to create a new channel segment $q_{\mathrm{L}}$ for that case are expected. The analysis of this fact for the propagation of upward connecting leaders is the subject of a future publication.

\section{Conclusion}

An improved physical leader propagation model is introduced in this paper. It differs from the existing models in such a way that the leader velocity and current are self-consistently computed based on the analysis of the corona charge required to create a new leader segment. It is shown that the charge per unit length of an upward leader cannot be assumed as a constant value equal to $50 \mu \mathrm{C} \mathrm{m}^{-1}$ as has been considered up to now. Since this parameter depends upon the energy available at the tip of the upward leader, its value depends upon the electrostatic conditions imposed by the descent of the downward leader. Therefore, the upward leader velocity and current are also affected by these conditions. This fact suggests that the upward leader velocity may change for different prospective return stroke peak currents of the downward leader and that it cannot be generalized.

\section{Acknowledgments}

The research work reported here is funded partly by the Swedish Research Council (Grant No G-EG/GU 1448-306), the Swedish Rescue Services Agency (Grant No 012-70392003) and a donation to the Uppsala University by John and Svea Andersson.

\section{References}

[1] Golde R H 1967 The lightning conductor J. Franklin Inst. 283 451-77

[2] Eriksson A J 1987 An improved electrogeometric model for transmission line shielding analysis IEEE Trans. Power Delivery PWDR-2 871-86

[3] Eriksson A J 1987 The incidence of lightning strikes to power lines IEEE Trans. Power Delivery PWDR-2 859-70

[4] Carrara G and Thione L 1979 Switching surge strength of large air gaps: a physical approach IEEE Trans. Power Appar. Syst. PAS-95 512-24

[5] Dellera L and Garbagnati E 1990 Lightning strike simulation by means of the leader progression Model: I. Description of the model and evaluation of free-standing structures IEEE Trans. Power Delivery PWRD-5 2009-23

[6] Dellera L and Garbagnati E 1990 Lightning strike simulation by means of the Leader Progression Model: II. Exposure and shielding failure evaluation of overhead lines with assessment of application graphs IEEE Trans. Power Delivery PWRD-5 2023-9

[7] Rizk F 1994 Modeling of lightning incidence to tall structures IEEE Trans. Power Delivery PWRD-9 172-93

[8] Rizk F 1990 Modeling of transmission lines: exposure to direct lightning strokes IEEE Trans. Power Delivery PWRD-5 1983-9

[9] Rizk F 1989 A model for switching impulse leader inception and breakdown of long air-gaps IEEE Trans. Power Delivery PWRD-4 596-606

[10] Rizk F 1989 Switching impulse strength of air insulation: leader inception criterion IEEE Trans. Power Delivery PWRD-4 2187-95 
[11] Yokoyaman S, Miyake M, Suzuki T and Kanao S 1990 Winter lightning on Japan sea coast-development of measuring system on progressin feature of lightning discharge IEEE Trans. Power Delivery PWRD-5 1418-25

[12] Yokoyama S, Asakawa A, Miyake K, Shindo T, Wakai T and Sakai T 2000 Leader and Return stroke velocity measurements using advanced measuring system on progressing feature of lightning discharge (ALPS) Proc. 25th Int. Conf. on Lightning Protection: ICLP 2000 (Greece) pp 66-71

[13] Kito Y, Horii K, Higashiyama Y and Nakamura K 1985 Optical aspects of winter lightning discharges triggered by the rocket-wire technique in Hukuriku district of Japan J. Geophys. Res. 90 6147-57

[14] Lalande P, Bondiou A, Laroche P, Eybert A, Berlandis J, Bador B, Bonamy A, Uman M and Rakov V 1998 Leader properties determined with triggered lightning techniques J. Geophys. Res. 103 14109-15

[15] Willet J C, Davis D A and Laroche P 1999 An experimental study of positive leaders initiating rocket-triggered lightning Atmos. Res. 51 189-219

[16] Laroche P, Eybert-Berard A, Barret L and Berlandis J P 1988 Observations of preliminary discharge initiating flashes triggred by the rocket and wire technique Proc. Int. Conf. Atmospheric Electricity (USA, 1988) pp 327-33

[17] Bondiou A and Gallimberti I 1994 Theoretical modeling of the development of the positive spark in long gaps J. Phys. D: Appl. Phys. 27 1252-66

[18] Goelian N, Lalande P, Bondiou-Clergerie A, Bacchiega G L, Gazzani A and Gallimberti I 1997 A simplified model for the simulation of positive-spark development in long air gaps J. Phys. D: Appl. Phys. 30 2441-52
[19] Becerra M, Cooray V 2006 A simplified physical model to determine the lightning upward connecting leader inception Trans. IEEE Power Delivery PWRD-21 897-908

[20] Becerra M, Cooray V 2006 Dynamic modelling of the lightning upward connecting leader inception 28th Int. Conf. on Lightning Protection: ICLP 2006 (Kanazawa Japan) to be presented

[21] Bondiou-Clergerie A, Lalande P, Laroche P, Willet J C, Davis D, Gallimberti I 1999 The inception phase of positive leaders in triggered lightning: comparison of modeling with experimental data ICAE 99: 11th Int. Conf. on Atmospheric Electricity, (Alabama, USA, June 1999)

[22] Gallimberti I 1972 The mechanism of long spark formation J. Physique Coll. (Suppl.) 40 193-250

[23] Singer H, Steinbigler H and Weiss P 1974 A charge simulation method for the calculation of high voltage fields IEEE Trans. Power Appar. Syst. PAS-93 1660-8

[24] Les Renardières Group 1977 Positive discharges in long air gaps-1975 results and conclusions Electra 53 31-132

[25] Les Renardières Group 1974 Research on long air gap discharges-1973 results Electra 35 47-155

[26] Les Renardières Group 1972 Research on long air gap discharges Electra 23 53-157

[27] Berger K 1967 Novel observations of lightning discharges: results of research on Mount San Salvatore J. Franklin Inst. 283 478-525

[28] Lalande P 1996 Study of the lightning stroke conditions on a grounded structure Doctoral Thesis A publication of Office National d'Etudes et de Recherches A'erospatiales (ONERA)

[29] Lalande P, Bondiou A, Bacchiega G and Gallimberti I 2002 Observations and modelling of lightning leaders C. R. Phys. 3 1375-92 